Archived version from NCDOCKS Institutional Repository http://libres.uncg.edu/ir/asu/

\title{
Appalacȟ̉an
}

$\overline{\text { B O O N E, N O R T H C A R O L I A }}$

\section{Effect Of Mixed Flavonoids, N-3 Fatty Acids, And Vitamin C On Oxidative Stress And Antioxidant Capacity Before And After Intense Cycling}

\author{
By: Steven R. McAnulty, David C. Nieman, Lisa S. McAnulty, Worley S. Lynch, \\ Fuxia Jin, and Dru A. Henson
}

\begin{abstract}
Consumption of plant flavonoids, antioxidants, and n-3 fatty acids is proposed to have many potential health benefits derived primarily through antioxidant and anti-inflammatory activities. This study examined the effects of $1,000 \mathrm{mg}$ quercetin $+1,000 \mathrm{mg}$ vitamin $C(Q C) ; 1,000 \mathrm{mg}$ quercetin, 1,000 $\mathrm{mg}$ vitamin $\mathrm{C}, 400 \mathrm{mg}$ isoquercetin, $30 \mathrm{mg}$ epigallocatechin gallate, and $400 \mathrm{mg} \mathrm{n}-3$ fatty acids (QFO); or placebo (P), taken each day for 2 wk before and during $3 \mathrm{~d}$ of cycling at $57 \% \mathrm{Wmax}$ for $3 \mathrm{hr}$, on plasma antioxidant capacity (ferric- reducing ability of plasma [FRAP], oxygen-radical absorbance capacity [ORAC]), plasma oxidative stress (F2-isoprostanes), and plasma quercetin and vitamin C levels. Thirty-nine athletes were recruited and random- ized to QC, QFO, or P. Blood was collected at baseline, after 2 wk supplementation, immediately postexercise, and $14 \mathrm{hr}$ postexercise. Statistical design used a 3 (groups) $\times 4$ (times) repeated-measures ANOVA with post hoc analyses. Plasma quercetin was significantly elevated in QC and QFO compared with P. Plasma F2- isoprostanes, FRAP, and vitamin $C$ were significantly elevated and ORAC significantly decreased immediately postexercise, but no difference was noted in the overall pattern of change. Post hoc analyses revealed that the QC and QFO groups did not exhibit a significant increase in F2-isoprostanes from baseline to immediately postexercise compared with $\mathrm{P}$. This study indicates that combining flavonoids and antioxidants with $n-3$ fatty acids is effective in reducing the immediate postexercise increase in F2-isoprostanes. Moreover, this effect occurs independently of changes in plasma antioxidant capacity.
\end{abstract}

McAnulty, Steven \& Nieman, David \& McAnulty, Lisa \& Lynch, Worley \& Jin, Fuxia \& Henson, Dru. (2011). Effect of Mixed Flavonoids, n-3 Fatty Acids, and Vitamin C on Oxidative Stress and Antioxidant Capacity Before and After Intense Cycling. International journal of sport nutrition and exercise metabolism. 21. 328-37. DOI: 10.1 123/ijsnem.21.4.328. Publisher version of record available at: https://journals.humankinetics.com/view/ journals/ijsnem/21/4/article-p328.xml 


\title{
Effect of Mixed Flavonoids, n-3 Fatty Acids, and Vitamin $\mathrm{C}$ on Oxidative Stress and Antioxidant Capacity Before and After Intense Cycling
}

\author{
Steven R. McAnulty, David C. Nieman, Lisa S. McAnulty, \\ Worley S. Lynch, Fuxia Jin, and Dru A. Henson
}

\begin{abstract}
Consumption of plant flavonoids, antioxidants, and n-3 fatty acids is proposed to have many potential health benefits derived primarily through antioxidant and anti-inflammatory activities. This study examined the effects of 1,000 mg quercetin +1,000 $\mathrm{mg}$ vitamin C (QC); 1,000 $\mathrm{mg}$ quercetin, 1,000 $\mathrm{mg}$ vitamin C, $400 \mathrm{mg}$ isoquercetin, $30 \mathrm{mg}$ epigallocatechin gallate, and $400 \mathrm{mg} \mathrm{n}-3$ fatty acids (QFO); or placebo (P), taken each day for $2 \mathrm{wk}$ before and during $3 \mathrm{~d}$ of cycling at $57 \% \mathrm{~W}_{\max }$ for $3 \mathrm{hr}$, on plasma antioxidant capacity (ferricreducing ability of plasma [FRAP], oxygen-radical absorbance capacity [ORAC]), plasma oxidative stress ( $\mathrm{F}_{2}$-isoprostanes), and plasma quercetin and vitamin $\mathrm{C}$ levels. Thirty-nine athletes were recruited and randomized to QC, QFO, or P. Blood was collected at baseline, after 2 wk supplementation, immediately postexercise, and $14 \mathrm{hr}$ postexercise. Statistical design used a 3 (groups) $\times 4$ (times) repeated-measures ANOVA with post hoc analyses. Plasma quercetin was significantly elevated in QC and QFO compared with P. Plasma $\mathrm{F}_{2-}$ isoprostanes, FRAP, and vitamin $\mathrm{C}$ were significantly elevated and ORAC significantly decreased immediately postexercise, but no difference was noted in the overall pattern of change. Post hoc analyses revealed that the $\mathrm{QC}$ and QFO groups did not exhibit a significant increase in $\mathrm{F}_{2}$-isoprostanes from baseline to immediately postexercise compared with $\mathrm{P}$. This study indicates that combining flavonoids and antioxidants with $\mathrm{n}-3$ fatty acids is effective in reducing the immediate postexercise increase in $\mathrm{F}_{2}$-isoprostanes. Moreover, this effect occurs independently of changes in plasma antioxidant capacity.
\end{abstract}

Keywords: exercise, quercetin, humans, $\mathrm{F}_{2}$-isoprostanes, FRAP, ORAC

Recently, there has been interest in the potential antioxidant and anti-inflammatory activities of plantbased compounds such as flavonoids (Manach, Hubert, Llorach, \& Scalbert, 2009; Manach, Williamson, Morand, Scalbert, \& Remesy, 2005). These compounds may have important health benefits and consequences, but human studies are limited regarding the compounds and even more so when used in conjunction with exercise (McAnulty et al., 2008; Nieman, Henson, Davis, et al., 2007). One of the most often-studied flavonoids is quercetin, which is found in abundance in red wine, onions, red grapes, apples, tea, broccoli, and other green leafy vegetables. Previous research has reported quercetin to display metal-chelating ability and high antioxidant capacity (Manach et al., 2009; Manach, Williamson, et al., 2005). Humans can absorb large amounts of quercetin, which has an estimated plasma half-life ranging from 3.5 to $28 \mathrm{hr}$ (Manach, Scalbert, Morand, Remesy, \& Jimenez,

S. McAnulty, Nieman, and Lynch are with the Dept. of Health, Leisure, and Exercise Science; L. McAnulty, the Dept. of Nutrition and Health Care Management; Jin, the Dept. of Chemistry; and Henson, the Dept. of Biology, Appalachian State University, Boone, NC.
2004). In addition, epidemiological studies indicate that elevated quercetin consumption is associated with reduced risk of heart disease, diabetes, and various types of cancer (Manach, Mazur, \& Scalbert, 2005; Seeram, Adams, Hardy, \& Heber, 2004).

Despite these proposed benefits and antioxidant actions, two articles (McAnulty et al., 2008; Nieman, Henson, Davis, et al., 2007) report that 3 weeks supplementation of $1,000 \mathrm{mg}$ quercetin per day failed to counter exercise-induced alterations in immunity, inflammation, and oxidative stress. One potential reason for a lack of positive outcomes with that study was that the quercetin supplements were ingested 10-24 hr before the completion of exercise. This may have caused too much of a delay in peak plasma quercetin metabolites, given the short half-life of quercetin (Manach et al., 2004). The short half-life suggests that a dose given within an hour of exercise may be more beneficial in reducing oxidative stress. Furthermore, it is known that coingestion of quercetin with other flavonoids such as catechins extends quercetin's bioavailability and potential bioactive effects (Manach \& Donovan, 2004; Manach et al., 2004; Manach, Williamson, et al., 2005). Catechins such as epigallocatechin-3-gallate (EGCG) have been shown in animal models to inhibit tumorigenesis during the initia- 
tion, promotion, and progression stages and to function as chemical antioxidants, which can quench free-radical species and chelate transition metals (Lambert \& Elias, 2010). It is not yet known whether ingestion of individual flavonoids results in blood concentrations capable of exerting systemic antioxidant effects in vivo (Guo, Kong, \& Meydani, 2009; Hagl et al., 2010; Halliwell, 2007, 2008). Therefore, coingestion of flavonoids and antioxidants might be beneficial in increasing absorption and extending bioavailability.

The n-3 fatty acids are of interest as a supplement and comprise a family of unsaturated fatty acids that mainly consists of $\alpha$-linoleic acid, eicosapentaenoic acid, and docosahexaenoic acid. These fatty acids have recently been touted to exert positive effects by reducing inflammation (Das, Ramos, \& Meguid, 2003). Although n-3 fatty acids have been heavily researched for potential benefit against certain diseases, questions remain about their abilities to decrease oxidative damage and inflammation when supplemented in conjunction with other antioxidants in athletes (Das et al., 2003; McAnulty et al., 2010).

Therefore, given the potential importance of flavonoid compounds, antioxidants, and n-3 fatty acids in reducing oxidative stress and inflammation and considering the limited information regarding ingestion of these compounds in human health and exercise, our study extended and improved on previous work conducted in endurance athletes. $\mathrm{F}_{2}$-isoprostanes were used as an indicator of oxidative stress. These compounds possess potent biological activity, mediate various aspects of oxidative stress, and have been shown to be accurate markers of lipid peroxidation (Morrow, 2005; Morrow \& Roberts, 1996, 1997). This study had two specific objectives. The first was to test the influence of a quercetin supplement, with or without EGCG, isoquercetin, n-3 fatty acids (eicosapentaenoic and docosahexaenoic acid), and vitamin $\mathrm{C}$, on $\mathrm{F}_{2}$-isoprostanes and plasma antioxidant capacity before and after a 3-day period of intensified exercise. The second objective was to determine whether $\mathrm{F}_{2}$-isoprostanes and antioxidant capacity would be differentially affected by either chronic or acute supplementation.

\section{Methods and Materials}

\section{Subjects}

Thirty-nine trained male $(n=32)$ and female $(n=7)$ cyclists were recruited through local and college cycling clubs and tested as experimental subjects. Written informed consent was obtained from each subject, and the experimental procedures were approved by the institutional review board of Appalachian State University.

\section{Supplementation}

The soft-chew supplements were designed by Quercegen Pharma (Newton, MA) and prepared by Nutravail
Technologies (Chantilly, VA). Each placebo (P) softchew contained brown-rice syrup, evaporated cane juice, carnuba wax, natural flavors, gelatin, soy lecithin, palm oil, glycerin, xylitol, mono- and diglyceride, corn starch, carrageenan, sucralose, and $20 \mathrm{kcal}$ energy, with citric acid to substitute for the taste of vitamin $\mathrm{C}$ and FD\&C yellow \#5 and FD\&C blue \#1 to substitute for the quercetin color. The cyclists were randomized to quercetin plus vitamin C (QC; $n=13)$, which was a chew with $250 \mathrm{mg}$ of quercetin, $250 \mathrm{mg}$ vitamin $\mathrm{C}$, $10 \mathrm{mg}$ niacin, and $200 \mu \mathrm{g}$ of folic acid; quercetin plus other bioactive substances (QFO; $n=14$ ), which contained the same ingredients as QC with an added 100 $\mathrm{mg}$ isoquercetin, $30 \mathrm{mg} \mathrm{EGCG}$, and $100 \mathrm{mg}$ of total omega-3 fatty acids (55 mg eicosapentaenoic acid, 45 $\mathrm{mg}$ of docosahexaenoic acid) each; or placebo (P; $n=$ 12), which was a chew with citric acid to substitute for the taste of vitamin $\mathrm{C}$. Under double-blind procedures, subjects ingested four QC, QFO, or P soft-chews per day (two in the morning at 7-8 a.m., two in the afternoon at 2 p.m.) for 14 days before and during a 3-day period of intensified exercise.

\section{Research Design}

Within 1 week before the start of supplementation, subjects reported to the university human performance laboratory for orientation and measurement of cardiorespiratory fitness. $\mathrm{VO}_{2 \max }$ was determined using a graded maximal protocol (25-W increase every 2 min starting at $150 \mathrm{~W}$ ) with the subjects using their own bicycles on CompuTrainer Pro Model 8001 trainers (RacerMate, Seattle, WA). $\mathrm{VO}_{2}$ and ventilation were measured using the MedGraphics CPX metabolic system (MedGraphics Corp., St. Paul, MN). Heart rate was measured using a chest heart-rate monitor (Polar Electro Inc., Woodbury, NY). Basic demographic and training data were obtained through a questionnaire. Body composition was measured by hydrostatic weighing.

Subjects agreed to avoid the use of large-dose vita$\mathrm{min} / \mathrm{mineral}$ supplements (above $100 \%$ of recommended dietary allowances), herbs, and medications known to affect immune function during the entire study. During orientation, they were instructed to follow a diet moderate in carbohydrate (using a food list) the weekend before and during the 3-day intensified exercise period. At 12-12:30 p.m. before each 3-hr cycling bout, subjects ingested a standardized liquid meal (Boost Plus, Mead Johnson Nutritionals, Evansville, IN) at an energy level of $63 \mathrm{~kJ} /$ $\mathrm{kg}(15 \mathrm{kcal} / \mathrm{kg})$. Boost Plus is a nutritionally complete, high-energy oral supplement with an energy density of 6.4 $\mathrm{kJ} / \mathrm{ml}(1.52 \mathrm{kcal} / \mathrm{ml})$ and $16 \%$ of energy as protein, $34 \%$ as fat, and $50 \%$ as carbohydrate. In quantities of $1,000 \mathrm{ml}$, Boost Plus exceeds daily-value recommendations for all major vitamins and minerals. No other food and beverage (other than water) was consumed from this meal until the end of the cycling bout. Subjects ingested 0.5-1 L water per hour of cycling. Before and after the first 14 days of supplementation, they provided blood samples at 8 a.m. 
after an overnight fast and avoiding exercise training for at least $12 \mathrm{hr}$ and before having ingested supplements. Subjects then came to the laboratory for 3 consecutive days and cycled from 3 to 6 p.m. at $\sim 57 \% \mathrm{~W}_{\max }$. During the test sessions, subjects cycled on their own bicycles on CompuTrainer Pro Model 8001 trainers with the exercise load set at $\sim 57 \% \mathrm{~W}_{\max }$. Metabolic measurements were taken every 30 min of cycling using the MedGraphics CPX metabolic system to verify workload. Blood samples were obtained immediately after completion of the third 3 -hr exercise bout (6 p.m.) and then again $14 \mathrm{hr}$ later (8 a.m., overnight fasted, and having avoided supplements since 2 p.m. the previous day).

\section{Blood Samples}

Blood samples were drawn from an antecubital vein with subjects in the supine position. Routine complete blood counts were performed using a Coulter STKS instrument (Coulter Electronics, Hialeah, FL) and provided hemoglobin and hematocrit for determination of plasma volume change using the method of Dill and Costill (1974). Other blood samples were centrifuged in sodium heparin or EDTA tubes, and plasma was aliquotted and then stored at $-80{ }^{\circ} \mathrm{C}$ before analysis. Figure 1 illustrates the supplement, exercise, and blood-draw schedule.

\section{Plasma Quercetin Analysis}

Total plasma quercetin (quercetin and its primary conjugates) was measured after solid-phase extraction via reversed-phase high-performance liquid chromatography with UV detection as previously described (Nieman, Henson, Grosset, et al., 2007). Quercetin conjugates were hydrolyzed by incubating $500-\mu$ l plasma aliquots with $10 \mu 110 \%$ DL-dithiothreitol solution, $50 \mu 10.58 \mathrm{M}$ acetic acid, and $50 \mu \mathrm{l}$ of a mixture of $\beta$-glucuronidase/ arylsulfatase and crude extract from Helix pomatia (Roche Diagnostics Corp., Indianapolis, IN) for $2 \mathrm{hr}$ at $37{ }^{\circ} \mathrm{C}$. Chromatographic analysis was performed using the Ultimate 3000 HPLC-PDA system (Dionex Corp., Sunnyvale, CA) with a Gemini C18 column (Phenomenex, Torrance, CA).

\section{Oxidative and Antioxidant Assays}

$\boldsymbol{F}_{2}$-Isoprostanes. Plasma $\mathrm{F}_{2}$-isoprostanes were determined using gas-chromatography mass spectrometry as previously described (Morrow \& Roberts, 1999). Briefly, free $\mathrm{F}_{2}$-isoprostanes were extracted from plasma added to a deuterated [2H4] $\mathrm{PGF}_{2}$ internal standard. The mixture was then added to a C18 Sep Pak column, followed by silica solid-phase extraction. $\mathrm{F}_{2}$-isoprostanes were converted to pentafluorobenzyl esters, subjected to thin-layer chromatography, and converted to trimethylsilyl ether derivatives. Samples were analyzed by negative-ion chemicalionization gas-chromatography mass spectrometry using an Agilent $6890 \mathrm{~N}$ gas chromatography interfaced to an Agilent 5975B inert MSD mass spectrometer (Agilent Technologies, Inc., Santa Clara, CA).

Ferric-Reducing Antioxidant Potential. Total plasma antioxidant potential was determined by a ferricreducing ability of plasma (FRAP) assay according to the methodology of Benzie and Strain (1996). The basis of this assay is that water-soluble reducing agents (antioxidants) in the plasma will reduce ferric ions to ferrous ions, which then react with an added chromogen. Samples and standards were analyzed in duplicate and expressed as ascorbate equivalents based on a physiologic ascorbate standard curve $(0-1,000 \mu \mathrm{mol})$. Intra- and interassay coefficients of variation were less than $5 \%$ and $7 \%$, respectively.

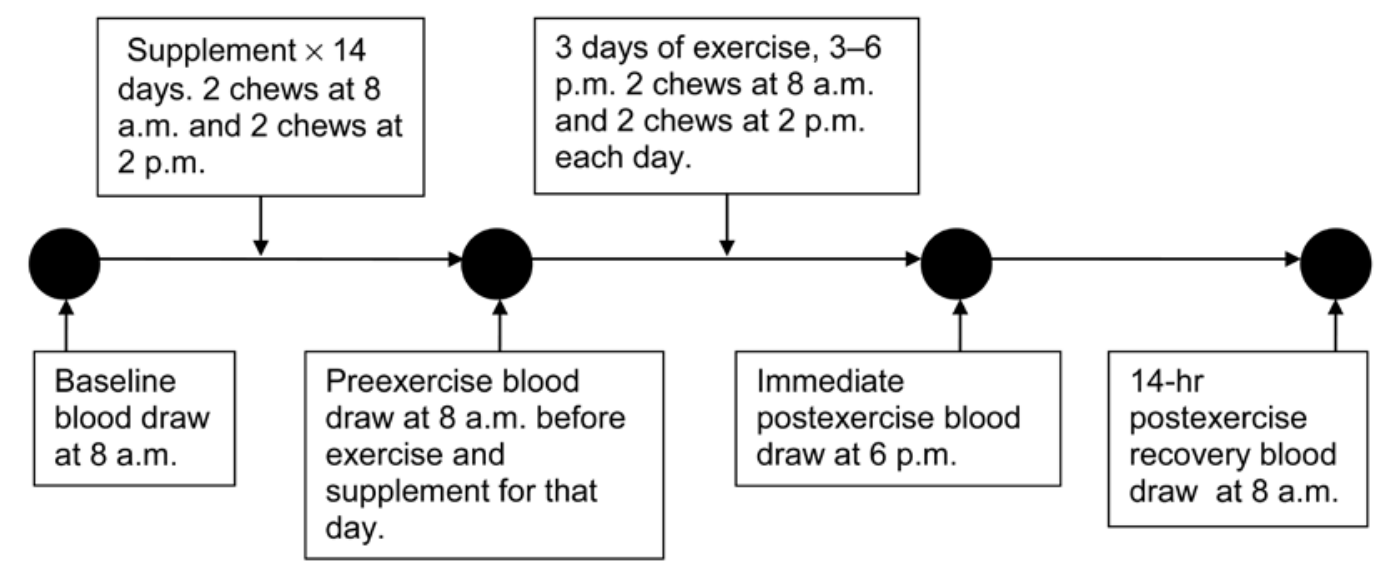

Figure 1 - Supplement, exercise, and blood-draw schedule. 
Oxygen-Radical Absorbance Capacity. The oxygenradical absorbance capacity (ORAC) assay was a modification of the methodologies of Ou, HampschWoodill, and Prior (2001). Trolox standards were made from a frozen Trolox stock solution, and working solution was made daily by thawing an aliquot. A serial dilution of the $50-\mu \mathrm{M}$ Trolox solution was made with working buffer solution to produce 25-, 12.5-, and 6.25- $\mu \mathrm{M}$ Trolox standards. A fluorescein (Aldrich Chemicals) working solution was made with phosphate buffer. Before use, the solution was incubated in a water bath at $37^{\circ} \mathrm{C}$ until thoroughly heated.

The 2,2'-azobis-2-methyl-propanimidamide, dihydrochloride (AAPH) solution was made by dissolving AAPH (Wako Chemical) into incubated phosphate buffer immediately before the start of the assay. The microplate was prepared and loaded in a forward-then-reverse order to avoid possible positional errors. The edge wells were left empty or blank (phosphate buffer working solution) to reduce the impact of "edge effect" on samples and standards, particularly from temperature effects on the outside wells. Twenty microliters of sample, blank, and Trolox standard solutions were pipetted into appropriate wells. Then, $200 \mu \mathrm{l}$ fluoroscein working solution were added to each well using an eight-channel micropipettor. A cover was placed on the microplate, and the plate and contents were incubated at $37^{\circ} \mathrm{C}$ for at least $20 \mathrm{~min}$. Then, $20 \mu \mathrm{AAPH}$ working solution were added using an eight-channel micropipettor as quickly as possible. Final ORAC values ( $\mu \mathrm{M} / \mathrm{L}$ Trolox) were made from the plate-reader-derived area under the curve. Then, the final $\mathrm{ORAC}_{\mathrm{FL}}$ values were calculated with a quadraticregression equation:

$$
x=-b \pm \sqrt{b^{2}-4 a c+4} c y
$$

Vitamin C. Vitamin C concentration of duplicate plasma samples was determined according to an adaptation of the methodology of Blake (2007). Briefly, a standard curve was developed using vitamin $\mathrm{C}$ standards in $10 \%$ TCA (Sigma, St. Louis, MO) over a concentration range of 0-25 $\mu \mathrm{M}$. Plasma samples were then centrifuged at $1,500 \mathrm{~g}$ for $15 \mathrm{~min}$ at $4{ }^{\circ} \mathrm{C}$, and the supernatant was removed and divided into duplicates of $0.5 \mathrm{ml}$ each. Then, $0.1 \mathrm{ml}$ of DTC solution was added to each standard and sample. The DTC solution consisted of 3.0 $\mathrm{g}$ of dinitrophenylhydrazine (DNPH; Sigma, St. Louis, MO), $0.4 \mathrm{~g}$ thiourea (Sigma), and $0.05 \mathrm{~g}$ copper sulfate pentahydrate (Sigma) in a final volume of $100 \mathrm{ml}$ with 9N sulfuric acid (Fisher Scientific, Pittsburgh, PA).

Samples and standards were gently vortexed, covered with aluminum foil, and placed in a water bath at $37^{\circ} \mathrm{C}$ in a dark room for $3 \mathrm{hr}$. Each tube was vortexed every $30 \mathrm{~min}$. Then, $750 \mu \mathrm{M}$ of $5 \%$ sulfuric acid were added to each tube and mixed well. The tubes were allowed to stand at room temperature in the dark for $1 \mathrm{hr}$ and were vortexed gently after $30 \mathrm{~min}$. The absorbance of each standard and sample was then read at $515 \mathrm{~nm}$, and the blank absorbance was subtracted. Sample concentrations were determined by a linear-regression line derived from a vitamin $\mathrm{C}$ standard curve.

\section{Statistical Analysis}

Data are expressed as $M \pm S D$. Data in Table 1 were compared between groups using one-way ANOVA. Data in Figures 2 through 6 were analyzed using a 3 (groups) $\times 4$ (time points) repeated-measures ANOVA with Tukey post hoc comparisons. Changes across the four time points were calculated and compared within groups. Significance for all comparisons was set at $p \leq .05$. This data analysis allowed group comparisons for three different effects: 2 weeks chronic supplementation (pre- and postsupplementation), acute postexercise changes, and 14-hr postexercise recovery.

\section{Results}

Subject characteristics for the 39 cyclists randomized to QC (11 men, 2 women), QFO (11 men, 3 women), and $\mathrm{P}$ (10 men, 2 women) and completing all phases of the study are summarized in Table 1. Outcome data for male and female cyclists did not differ significantly and are presented together for each group. No significant differences were found between groups for age, body composition, training history, or maximal performance measures. Plasma volume change did not differ between groups postexercise on the third day and averaged less than $2 \%$ as a result of ingestion of $0.5-1.0 \mathrm{~L}$ water per hour of exercise (data not shown).

Table 1 Subject Characteristics $(N=39), M \pm S D$

\begin{tabular}{lcccc}
\hline Variable & $\mathbf{P}(\boldsymbol{n}=\mathbf{1 2})$ & $\mathbf{Q F O}(\boldsymbol{n}=\mathbf{1 4})$ & $\mathbf{Q C}(\boldsymbol{n}=\mathbf{1 3})$ & $\boldsymbol{p}$ \\
\hline Age (years) & $26.3 \pm 1.7$ & $28.1 \pm 2.8$ & $26.8 \pm 2.6$ & .747 \\
Weight $(\mathrm{kg})$ & $72 \pm 4$ & $71.8 \pm 2.9$ & $72.6 \pm 3.8$ & .920 \\
Body fat $(\%)$ & $11.4 \pm 1.7$ & $12.2 \pm 1.7$ & $14.1 \pm 1.6$ & .486 \\
Training $(\mathrm{km} / \mathrm{week})$ & $308 \pm 40$ & $248 \pm 27$ & $227 \pm 25$ & .295 \\
Maximal $\mathrm{VO}_{2}\left(\mathrm{ml} \cdot \mathrm{kg}^{-1} \cdot \mathrm{min}^{-1}\right)$ & $60.2 \pm 1.8$ & $60.3 \pm 2.2$ & $61.4 \pm 2.3$ & .796 \\
Maximal power $(\mathrm{W})$ & $308 \pm 12$ & $321 \pm 16$ & $301 \pm 17$ & .553 \\
Maximal heart rate (beats/min) & $189 \pm 3$ & $184 \pm 2$ & $187 \pm 2$ & .352 \\
\hline
\end{tabular}

Note. $\mathrm{P}=$ placebo; $\mathrm{QFO}=$ quercetin, flavonoids, and omega- 3 fatty acids; $\mathrm{QC}=$ quercetin and vitamin $\mathrm{C} ; \mathrm{VO}_{2}=$ volume of oxygen consumption. 
aPlacebo $\quad$ Quercetin Quercetin-EGCG

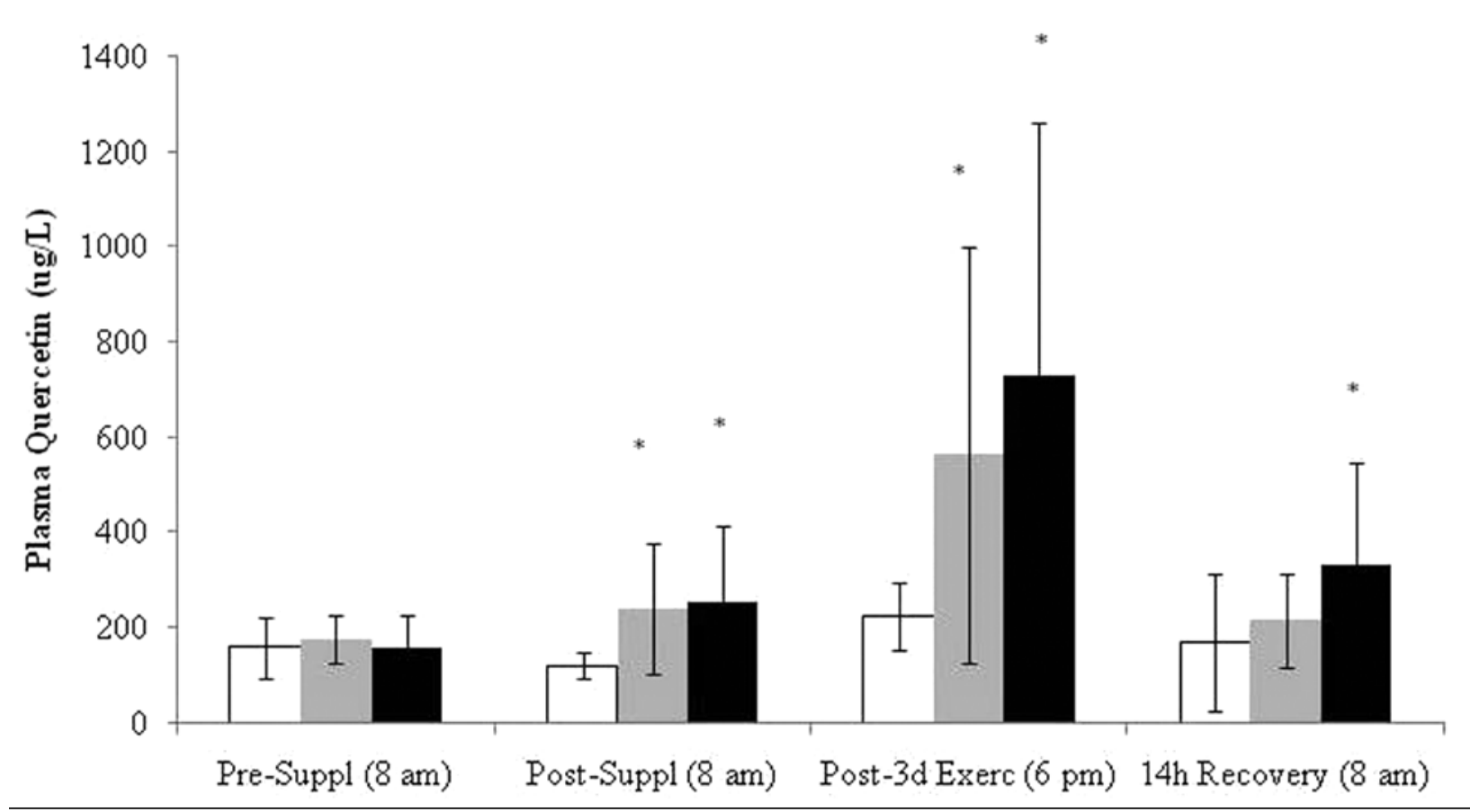

Figure 2 - Plasma quercetin concentrations in placebo $(n=12)$, quercetin $(n=13)$, and quercetin plus EGCT $(n=14)$ groups presupplement, postsupplement, after 3 days of exercise, and $14 \mathrm{hr}$ recovery, $M \pm S D$. Treatment $\times$ Time interaction $p=.008$. *Significantly different from placebo $(p \leq .05)$.

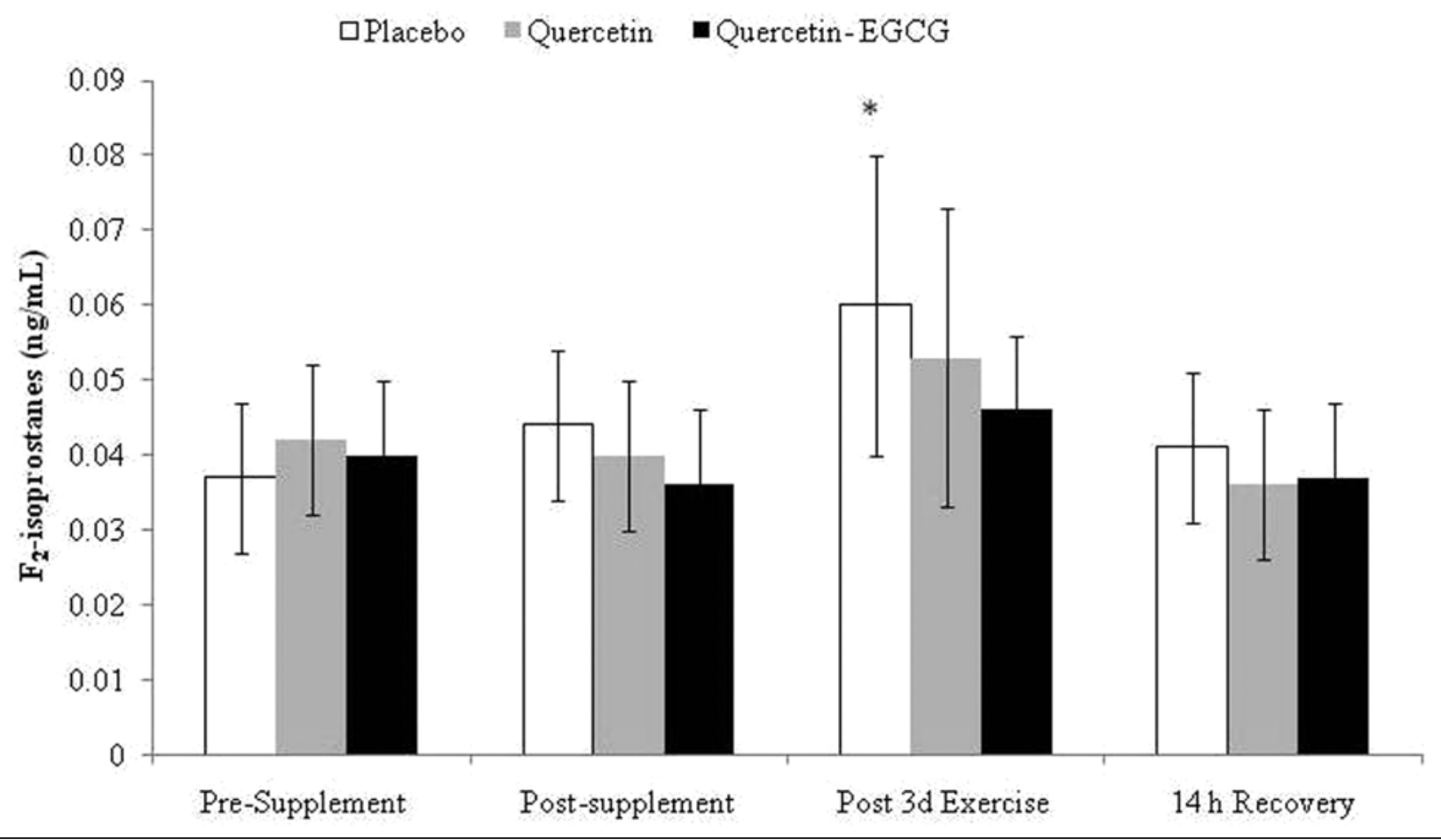

Figure 3 - Plasma $\mathrm{F}_{2}$-isoprostane values in placebo $(n=12)$, quercetin $(n=13)$, and quercetin plus EGCT $(n=14)$ groups presupplement, postsupplement, after 3 days of exercise, and $14 \mathrm{hr}$ recovery, $M \pm S D$. Main effects were treatment $(p=.497)$, time $(p$ $\leq .001)$, and Treatment $\times$ Time interaction $(p=.144)$. $*$ Significantly different from presupplement $(p \leq .01)$. 


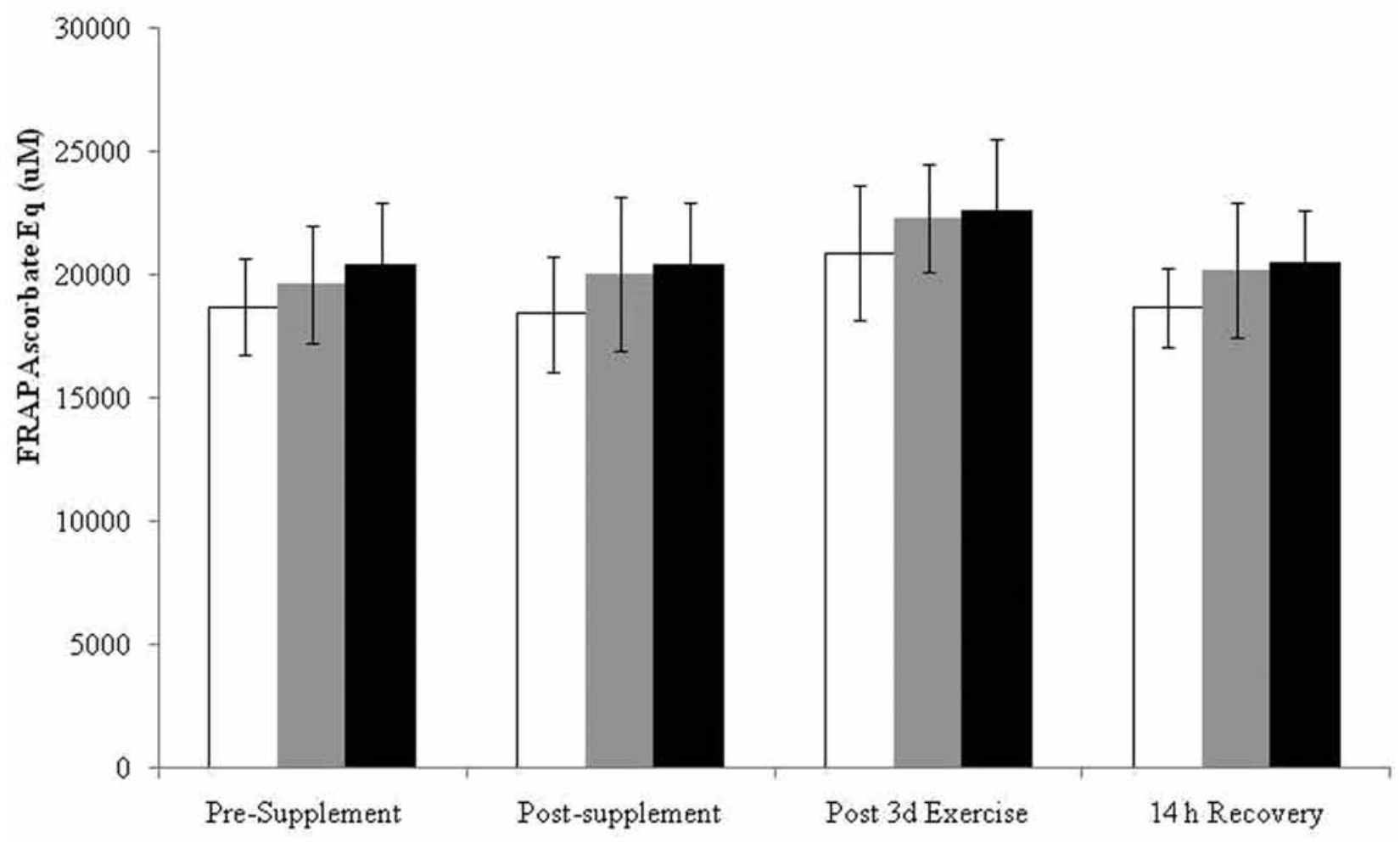

Figure 4 - Plasma ferric-reducing ability of plasma (FRAP) values in placebo $(n=12)$, quercetin $(n=13)$, and quercetin plus EGCT $(n=14)$ groups presupplement, postsupplement, after 3 days of exercise, and $14 \mathrm{hr}$ recovery, $M \pm S D$. Main effects were treatment $(p=.101)$, time $(p \leq .001)$, and Treatment $\times$ Time interaction $(p=.917)$.

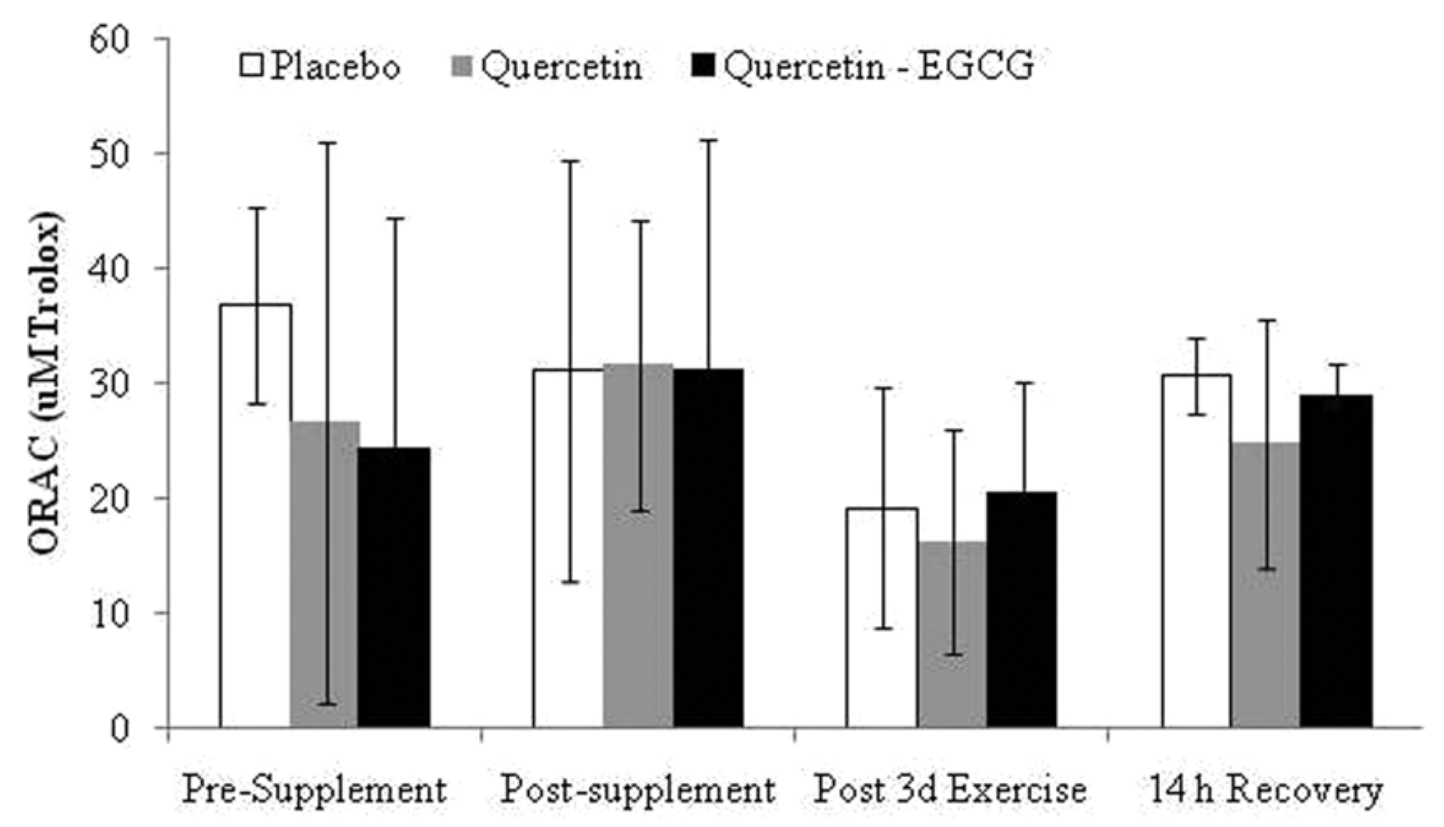

Figure 5 - Plasma oxygen-radical absorbance capacity (ORAC) values in placebo $(n=12)$, quercetin $(n=13)$, and quercetin plus EGCT $(n=14)$ groups presupplement, postsupplement, after 3 days of exercise, and $14 \mathrm{hr}$ recovery, $M \pm S D$. Main effects were treatment $(p=.104)$, time $(p=.024)$, and Treatment $\times$ Time interaction $(p=.351)$. 


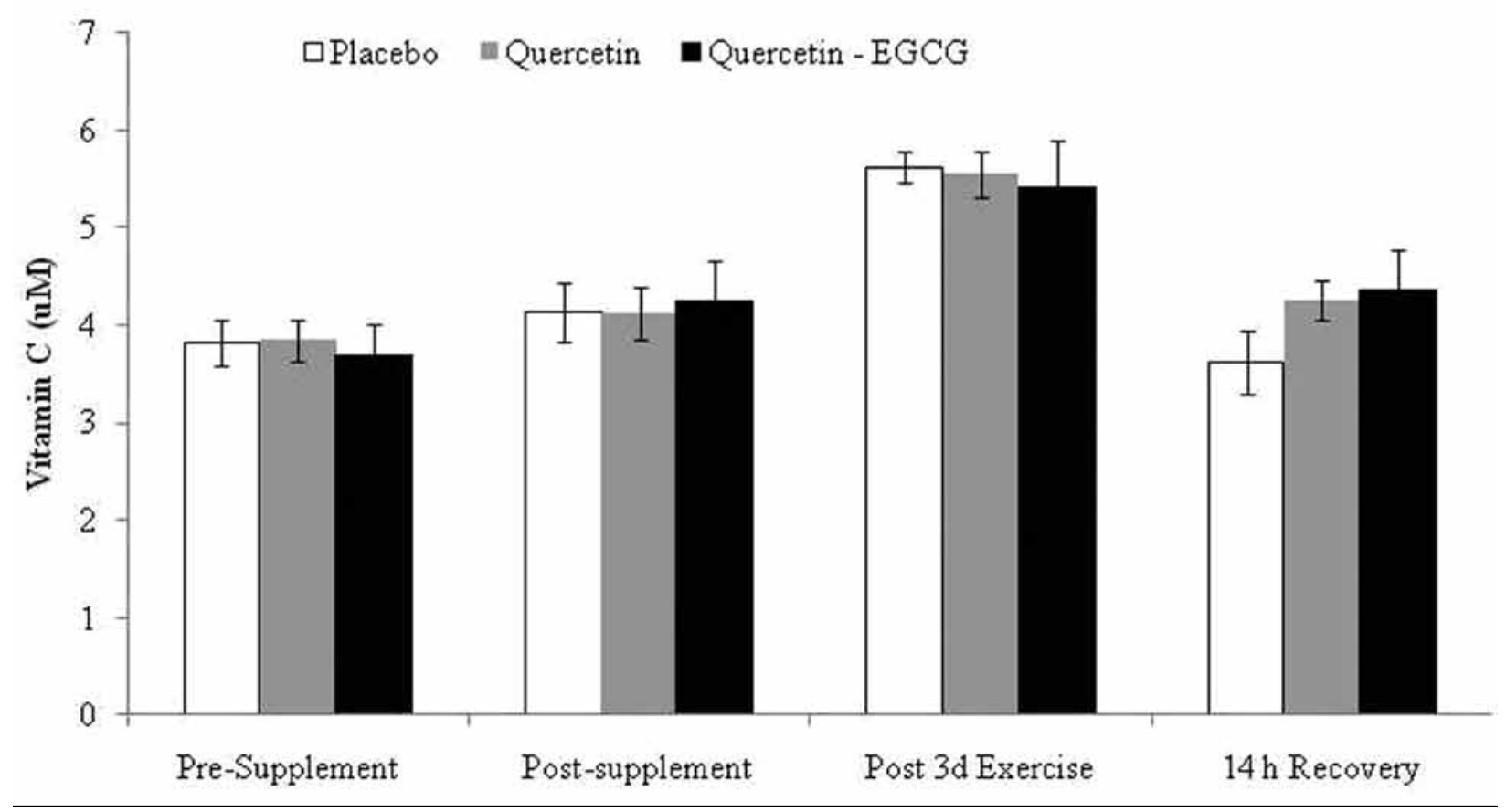

Figure 6 - Plasma vitamin C values in placebo $(n=12)$, quercetin $(n=13)$, and quercetin plus EGCT $(n=14)$ groups presupplement, postsupplement, after 3 days of exercise, and $14 \mathrm{hr}$ recovery, $M \pm S D$. Main effects were treatment $(p=.149)$, time $(p \leq .000)$, and Treatment $\times$ Time interaction $(p=.203)$.

After 2 weeks of consuming two soft-chew supplements at 8 a.m. and 2 p.m. each day, overnight-fasted plasma quercetin levels were $36 \%$ and $62 \%$ above presupplementation levels in the QC and QFO groups, respectively, compared with a $24 \%$ decrease in $\mathrm{P}$ (Figure 2). After the third day of exercise, plasma quercetin levels at 6 p.m. (i.e., $4 \mathrm{hr}$ after the last supplement dose) were $221 \%$ and $364 \%$ above presupplementation levels in the QC and QFO groups, respectively. At 14-hr postexercise, a significant $111 \%$ increase in plasma quercetin levels was measured in the QFO group.

$\mathrm{F}_{2}$-isoprostanes were elevated as a result of exercise (time effect $p \leq .001$ ), but no overall treatment effects were found. However, after post hoc analysis between baseline and immediately postexercise, groups QC and QFO did not exhibit a significant increase in $\mathrm{F}_{2}-$ isoprostanes compared with P. Postexercise increases were QFO 15\%, QC 26\%, and P 62\% (Figure 3). FRAP was also elevated as a result of exercise (time effect $p \leq$ .001 ; Figure 4 ), whereas ORAC values declined postexercise (time effect $p=.017$; Figure 5). However, no overall treatment or post hoc effects or differences were found for FRAP or ORAC (Figures 4 and 5, respectively).

Plasma vitamin $C$ values exhibited an increase after exercise (time effect, $p \leq .001$ ), but no overall treatment effects were found. Exercise caused a 35\% increase in P, $34 \%$ increase in QA, and 28\% increase in QOF (Figure 6 ). In addition, a significant but weak inverse correlation ( $p=.002, r=-.239)$ was found between $\mathrm{F}_{2}$-isoprostanes and vitamin $\mathrm{C}$, but no other significant correlations were observed.

\section{Discussion}

The main outcome found in this investigation was that supplementation with combinations of quercetin, isoquercetin, vitamin C, n-3 fatty acids, and EGCG (catechin) prevented significant increases in $\mathrm{F}_{2}$-isoprostanes immediately after 3 days of strenuous cycling versus placebo. This occurred despite a demonstrated lack of change in antioxidant status. It is not possible from our investigation to determine precisely which agent or combinations were responsible for the $\mathrm{F}_{2}$-isoprostane reduction, but this should be systematically investigated in the future. Although the complete ramifications are not known concerning exercise, it is known that $\mathrm{F}_{2}$-isoprostanes possess potent biological activity and mediate various aspects of oxidative stress. A number of studies have shown them to be accurate markers of lipid peroxidation and to be associated with several human diseases such as obesity, diabetes, and atherosclerosis (Morrow, 2005; Morrow \& Roberts, 1996, 1997). There was no effect of the chronic 14 days of supplementation on $\mathrm{F}_{2}$-isoprostanes or any antioxidant measure. The postexercise suppression of $\mathrm{F}_{2}$-isoprostanes in the QA and QFO groups after 3 days of exercise must have resulted from the effects of acute ingestion. This brings out the possible importance of the half-life in the blood of the flavonoids and the importance of maximum blood concentrations of flavonoids and n-3 fatty acids when exercise is conducted.

Because we observed no changes in antioxidant status, it is possible that other mechanisms such as inflammatory pathways might have been involved in affecting 
$\mathrm{F}_{2}$-isoprostane production. Loke et al. (2008) have suggested that depending on the type of conjugation (sulfate vs. gluconate) and the hydroxyl group site of the conjugation, anti-inflammatory effects of polyphenols may be maintained while antioxidant effects are lost. Davi, Falco, and Patrono (2004) reported that the adverse effects of inflammatory states may be mediated, at least in part, through enhanced lipid peroxidation and production of $\mathrm{F}_{2}$-isoprostanes. Many other studies have examined various disease states and found clear associations between elevated $\mathrm{F}_{2}$-isoprostanes and inflammatory states (Cracowski, Bonaz, Bessard, Bessard, Anglade, \& Fournet, 2002; Jialal, Devaraj, \& Venugopal, 2002; Zanconato et al., 2004). Exercise has also been shown to induce an inflammatory and oxidative state (Nieman et al., 2005; Nieman, Davis, et al., 2003; Nieman, Dumke, et al., 2003). The presence of n-3 fatty acids in the QFO group may have reduced inflammation by competition with arachidonic acid as a precursor in the cyclo-oxygenase pathway. This results in modification of lipid-derived eicosanoids and a reduction of inflammatory cytokines, which most likely occur by effects on transcription factors that regulate inflammatory gene expression (Calder, 2009; Calder \& Yaqoob, 2009). Because both QA and $\mathrm{QFO}$ caused suppression of $\mathrm{F}_{2}$-isoprostanes, it appears that quercetin and vitamin $\mathrm{C}$ are also effective antioxidant agents, and the addition of n-3 fatty acids and other mixed flavonoids increases the effectiveness of the supplement ( $26 \%$ vs. $15 \%$ increase in $\mathrm{F}_{2}$-isoprostanes, respectively). The origin of $\mathrm{F}_{2}$-isoprostanes detected in plasma remains controversial in that a multitude of tissues such as skeletal muscle, red blood cells, and plasma can produce $\mathrm{F}_{2}$-isoprostanes (Nikolaidis \& Jamurtas, 2009).

We also observed that quercetin supplementation dramatically increases circulating plasma values of quercetin. However, in contrast to our hypothesis, this plasma increase did not affect plasma antioxidant capacity. This finding is counter to previous investigations demonstrating the efficacy of quercetin as an in vitro antioxidant (Manach et al., 2004; Manach et al., 1998). There have been few human intervention studies with quercetin, but Williamson and Manach (2005) reviewed human studies using doses from $21 \mathrm{mg}$ to $1,000 \mathrm{mg}$ of quercetin, all of which exhibited at least some beneficial effects, although those investigations did not use $\mathrm{F}_{2}$-isoprostanes as an indicator of oxidative stress. However, McAnulty et al. (2008) found that $1,000 \mathrm{mg}$ quercetin per day for 6 weeks did not suppress $\mathrm{F}_{2}$-isoprostanes or increase plasma antioxidant capacity. This lends further support to the notion that combinations of polyphenols (as found in the human diet) or n-3 fatty acid supplements might be necessary to exert biological effects.

Given that there were no treatment effects for FRAP and ORAC, our data indicate that the potential health benefits attributed to quercetin ingestion might occur through mechanisms beyond that related to strict antioxidant function. Williams, Spencer, and Rice-Evans (2004) suggested that flavonoid metabolites do not act as conventional hydrogen-donating antioxidants but modulate actions of protein- and lipid-kinase-signaling pathways, which result ultimately in modulation of gene expression. However, Manach et al. (2004; 1998) found that the conjugated metabolites of quercetin still exerted antioxidant properties, albeit at about $50 \%$ of the aglycone form. Quercetin exhibits powerful antioxidant activity in vitro because of the presence of a 3,4 B-ring hydroxyl group configuration. During quercetin metabolism in humans, substitution of the 3,4 B-ring hydroxyl groups with methyl or glycosyl groups abolishes a large amount of the free-radical-scavenging ability (Manach et al., 2004; 1998) and is likely the reason for lack of effect on the FRAP and ORAC assays. Galleano, Verstraeten, Oteiza, and Fraga (2010) suggest that the actual concentrations reached by flavonoids in most animal and human tissues after dietary ingestion are incompatible with the kinetic requirements necessary to reach reaction rates of physiological relevance and that other mechanisms are responsible for reducing oxidative stress and inflammation, such as interactions with membrane lipids and protein-flavonoid interactions. Our results indicate that the antioxidant capacity of quercetin and blood plasma is not additive in any form that contributes to preservation of reducing compounds able to demonstrate results with the FRAP or ORAC assays.

Plasma antioxidant capacity increased significantly as a result of exercise in the case of the FRAP assay but as previously stated was not affected by any of the supplement combinations. This increase in plasma antioxidant capacity detected by the FRAP assay most likely represents urate and ascorbate released into the blood during exercise (McAnulty et al., 2007; Yanai \& Morimoto, 2004). Conversely, plasma ORAC values declined significantly and similarly between treatments after exercise, suggesting depletion of one or more antioxidants. Briviba et al. (2007) found no significant changes in ORAC after bouts of acute ultraendurance exercise. In contrast, Neubauer et al. (2010) found ORAC to be significantly increased immediately after an Ironman triathlon. The uniqueness of the ORAC assay versus other antioxidant assays is that total antioxidant capacity of a sample is estimated by taking an oxidation reaction to completion. ORAC measures all the nonprotein antioxidants, which include alpha-tocopherol, glutathione, vitamin $\mathrm{C}$, beta-carotene, uric acid, and bilirubin. In this regard, it is more of a comprehensive antioxidant measurement encompassing multiple antioxidants (Cao, Verdon, Wu, Wang, \& Prior, 1995). These results confirm that the total antioxidant capacity of blood plasma is complex and highly regulated and that using different antioxidant assays will result in different outcomes after exercise. Because each assay measures a different antioxidant fraction (water and lipid soluble, respectively), one should be aware of this when using these assays to make assessments in exercise studies. FRAP and ORAC results were not correlated, and plasma vitamin $C$ values were also not correlated with either antioxidant measure.

In summary, supplementation with combinations of quercetin, vitamin C, isoquercetin, EGCG catechin, and 
n-3 fatty acids for 2 weeks reduced $\mathrm{F}_{2}$-isoprostanes immediately postexercise from baseline compared with placebo. However, despite the reduction in $\mathrm{F}_{2}$-isoprostanes, the supplementation did not alter plasma antioxidant capacity, which implies that quercetin and other flavonoids exert beneficial health effects from mechanisms unrelated to antioxidant functions.

\section{Acknowledgments}

This study was funded by a research grant from Quercegen Pharma, Newton, MA.

\section{References}

Benzie, I.F., \& Strain, J.J. (1996). The ferric reducing ability of plasma (FRAP) as a measure of "antioxidant power": The FRAP assay. Analytical Biochemistry, 239, 70-76.

Blake, C.J. (2007). Analytical procedures for water-soluble vitamins in foods and dietary supplements: A review. Analytical and Bioanalytical Chemistry, 389, 63-76.

Briviba, K., Stracke, B.A., Rufer, C.E., Watzl, B., Weibel, F.P., \& Bub, A. (2007). Effect of consumption of organically and conventionally produced apples on antioxidant activity and DNA damage in humans. Journal of Agricultural and Food Chemistry, 55, 7716-7721.

Calder, P.C. (2009). Polyunsaturated fatty acids and inflammatory processes: New twists in an old tale. Biochimie. Advance online publication.

Calder, P.C., \& Yaqoob, P. (2009). Omega-3 polyunsaturated fatty acids and human health outcomes. BioFactors (Oxford, England), 35, 266-272.

Cao, G., Verdon, C.P., Wu, A.H., Wang, H., \& Prior, R.L. (1995). Automated assay of oxygen radical absorbance capacity with the COBAS FARA II. Clinical Chemistry, $41,1738-1744$.

Cracowski, J.L., Bonaz, B., Bessard, G., Bessard, J., Anglade, C., \& Fournet, J. (2002). Increased urinary F2-isoprostanes in patients with Crohn's disease. The American Journal of Gastroenterology, 97, 99-103.

Das, U.N., Ramos, E.J., \& Meguid, M.M. (2003). Metabolic alterations during inflammation and its modulation by central actions of omega- 3 fatty acids. Current Opinion in Clinical Nutrition and Metabolic Care, 6, 413-419.

Dill, D.B., \& Costill, D.L. (1974). Calculation of percentage changes in volumes of blood, plasma, and red cells in dehydration. Journal of Applied Physiology, 37, 247-248.

Galleano, M., Verstraeten, S.V., Oteiza, P.I., \& Fraga, C.G. (2010). Antioxidant actions of flavonoids: Thermodynamic and kinetic analysis. Archives of Biochemistry and Biophysics, 501, 23-30.

Guo, W., Kong, E., \& Meydani, M. (2009). Dietary polyphenols, inflammation, and cancer. Nutrition and Cancer, 61, 807-810.

Hagl, S., Deusser, H., Soyalan, B., Janzowski, C., Will, F., Dietrich, H., . . \& \& Richling, E. (2010). Colonic availability of polyphenols and D-(-)-quinic acid after apple smoothie consumption. Molecular Nutrition and Food Research. Advance online publication.
Halliwell, B. (2007). Dietary polyphenols: Good, bad, or indifferent for your health? Cardiovascular Research, 73, 341-347.

Halliwell, B. (2008). Are polyphenols antioxidants or prooxidants? What do we learn from cell culture and in vivo studies? Archives of Biochemistry and Biophysics, 476, 107-112.

Jialal, I., Devaraj, S., \& Venugopal, S.K. (2002). Oxidative stress, inflammation, and diabetic vasculopathies: The role of alpha tocopherol therapy. Free Radical Research, 36, 1331-1336.

Lambert, J.D., \& Elias R.J. (2010). The antioxidant and prooxidant activities of green tea polyphenols: A role in cancer prevention. Archives of Biochemistry and Biophysics, 501, 65-72.

Loke, W.M., Proudfoot, J.M., Stewart, S., McKinley, A.J., Needs, P.W., Kroon, P.A., . . Kroft, K.D. (2008). Metabolic transformation has a profound effect on anti-inflammatory activity of flavonoids such as quercetin: Lack of association between antioxidant and lipoxygenase inhibitory activity. Biochemical Pharmacology, 75, 1045-1053.

Manach, C., \& Donovan, J.L. (2004). Pharmacokinetics and metabolism of dietary flavonoids in humans. Free Radical Research, 38, 771-785.

Manach, C., Hubert, J., Llorach, R., \& Scalbert, A. (2009). The complex links between dietary phytochemicals and human health deciphered by metabolomics. Molecular Nutrition \& Food Research, 53, 1303-1315.

Manach, C., Mazur, A., \& Scalbert, A. (2005). Polyphenols and prevention of cardiovascular diseases. Current Opinion in Lipidology, 16, 77-84.

Manach, C., Morand, C., Crespy, V., Demigne, C., Texier, O., Regerat, F., \& Remesy, C. (1998). Quercetin is recovered in human plasma as conjugated derivatives which retain antioxidant properties. FEBS Letters, 426, 331-336.

Manach, C., Scalbert, A., Morand, C., Remesy, C., \& Jimenez, L. (2004). Polyphenols: Food sources and bioavailability. The American Journal of Clinical Nutrition, 79, 727-747.

Manach, C., Williamson, G., Morand, C., Scalbert, A., \& Remesy, C. (2005). Bioavailability and bioefficacy of polyphenols in humans. I. Review of 97 bioavailability studies. The American Journal of Clinical Nutrition, 81, 230S-242S.

McAnulty, S.R., Hosick, P.A., McAnulty, L.S., Quindry, J.C., Still, L., Hudson, M.B., . . . Austin, M.D. (2007). Effect of pharmacological lowering of plasma urate on exerciseinduced oxidative stress. Applied Physiology, Nutrition, and Metabolism, 32, 1148-1155.

McAnulty, S.R., McAnulty, L.S., Nieman, D.C., Quindry, J.C., Hosick, P.A., Hudson, M.H., . . DiBarnardi, A. (2008). Chronic quercetin ingestion and exercise-induced oxidative damage and inflammation. Applied Physiology, Nutrition, and Metabolism, 33, 254-262.

McAnulty, S.R., Nieman, D.C., Fox-Rabinovich, M., Duran, V., McAnulty, L.S., Henson, D.A., . . L Landram, M.J. (2010). Effect of n-3 fatty acids and antioxidants on oxidative stress after exercise. Medicine and Science in Sports and Exercise, 42, 1704-1711. 
Morrow, J.D. (2005). Quantification of isoprostanes as indices of oxidant stress and the risk of atherosclerosis in humans. Arteriosclerosis, Thrombosis, and Vascular Biology, 25, 279-286.

Morrow, J.D., \& Roberts, L.J. (1996). The isoprostanes. Current knowledge and directions for future research. Biochemical Pharmacology, 51, 1-9.

Morrow, J.D., \& Roberts, L.J. (1997). The isoprostanes: Unique bioactive products of lipid peroxidation. Progress in Lipid Research, 36, 1-21.

Morrow, J.D., \& Roberts, L.J. (1999). Mass spectrometric quantification of $\mathrm{F}_{2}$-isoprostanes in biological fluids and tissues as measure of oxidant stress. Methods in Enzymology, 300, 3-12.

Neubauer, O., Reichhold, S., Nics, L., Hoelzl, C., Valentini, J., Stadlmayr, B., . . W Wagner, K.H. (2010). Antioxidant responses to an acute ultra-endurance exercise: Impact on DNA stability and indications for an increased need for nutritive antioxidants in the early recovery phase. The British Journal of Nutrition, 104, 1129-1138.

Nieman, D.C., Davis, J.M., Henson, D.A., Gross, S.J., Dumke, C.L., Utter, A.C., . . . Triplett, N.T. (2005). Muscle cytokine mRNA changes after $2.5 \mathrm{~h}$ of cycling: Influence of carbohydrate. Medicine and Science in Sports and Exercise, 37, 1283-1290.

Nieman, D.C., Davis, J.M., Henson, D.A., Walberg-Rankin, J., Shute, M., Dumke, C.L., . . McAnulty, L.S. (2003). Carbohydrate ingestion influences skeletal muscle cytokine mRNA and plasma cytokine levels after a 3-h run. Journal of Applied Physiology (Bethesda, Md.), 94, 1917-1925.

Nieman, D.C., Dumke, C.I., Henson, D.A., McAnulty, S.R., McAnulty, L.S., Lind, R.H., \& Morrow, J.D. (2003). Immune and oxidative changes during and following the Western States Endurance Run. International Journal of Sports Medicine, 24, 541-547.

Nieman, D.C., Henson, D.A., Davis, J.M., Angela, M.E., Jenkins, D.P., Gross, S.J., . . Mayer, E.P. (2007). Quercetin's influence on exercise-induced changes in plasma cytokines and muscle and leukocyte cytokine mRNA. Journal of Applied Physiology (Bethesda, Md.), 103, 1728-1735.

Nieman, D.C., Henson, D.A., Gross, S.J., Jenkins, D.P., Davis, J.M., Murphy, E.A., . . Mayer, E.P. (2007). Quercetin reduces illness but not immune perturbations after intensive exercise. Medicine and Science in Sports and Exercise, 39, 1561-1569.

Nikolaidis, M.G., \& Jamurtas, A.Z. (2009). Blood as a reactive species generator and redox status regulator during exercise. Archives of Biochemistry and Biophysics, 490, 77-84.

Ou, B., Hampsch-Woodill, M., \& Prior, R.L. (2001). Development and validation of an improved oxygen radical absorbance capacity assay using fluorescein as the fluorescent probe. Journal of Agricultural and Food Chemistry, 49, 4619-4626.

Seeram, N.P., Adams, L.S., Hardy, M.L., \& Heber, D. (2004). Total cranberry extract versus its phytochemical constituents: Antiproliferative and synergistic effects against human tumor cell lines. Journal of Agricultural and Food Chemistry, 52, 2512-2517.

Williams, R.J., Spencer, J.P., \& Rice-Evans, C. (2004). Flavonoids: Antioxidants or signalling molecules? Free Radical Biology \& Medicine, 36, 838-849.

Williamson, G., \& Manach, C. (2005). Bioavailability and bioefficacy of polyphenols in humans. II. review of 93 intervention studies. American Journal of Clinical Nutrition, 81, 243S-255S.

Yanai, H., \& Morimoto, M. (2004). Effect of ascorbate on serum lipids and urate metabolism during exhaustive training. Clinical Science (London, England), 106, 107-109.

Zanconato, S., Carraro, S., Corradi, M., Alinovi, R., Pasquale, M.F., Piacentini, G., . . Baraldi, E. (2004). Leukotrienes and 8-isoprostane in exhaled breath condensate of children with stable and unstable asthma. The Journal of Allergy and Clinical Immunology, 113, 257-263. 